\title{
Over-Expressed Bcl-2 Cannot Suppress Apoptosis via the Mitochondria in Buprenorphine Hydrochloride-Treated NG108-15 Cells
}

\author{
Fumihiko Kugawa, ${ }^{* a}$ Maiko Nakamura, ${ }^{a}$ Akemichi Ueno, ${ }^{b}$ and Masatada Aoki ${ }^{a}$ \\ ${ }^{a}$ Department of Biological Pharmaceutical Sciences, College of Pharmacy, Nihon University; 7-7-1 Narashino-dai, \\ Funabashi, Chiba 274-8555, Japan: and ${ }^{b}$ Department of Molecular Biology, Institute of Health Biosciences, The \\ University of Tokushima Graduate School; 3-18-15 Kuramoto-cho, Tokushima, Tokushima 770-8504, Japan. \\ Received March 9, 2004; accepted July 5, 2004; published online July 6, 2004
}

We previously reported that the morphine alkaloid derivative buprenorphine hydrochloride (Bph) induces rapid apoptosis in NG108-15 nerve cells accompanied by the activation of caspase-3. Here, we found this kind of apoptosis was also accompanied by rapid loss of the mitochondrial membrane potential, followed by the efflux of cytochrome $c$ from the mitochondria to the cytosol and the activation of caspases- 9 and $\mathbf{- 3}$. Together, these results strongly suggested the Bph death signal was routed through the mitochondrial pathway in NG108-15 cells. In these cells, serum-starvation induces a different apoptosis, which we exploited to investigate Bcl-2's role as an apoptosis inhibitor. We made an NG108-15 transfectant, Bcl-2(P2), that stably expressed human Bcl-2, and used it to test Bcl-2's effect on the serum-starvation-induced apoptosis in NG108-15 cells. Cell viability, DNA-ladder formation, and efflux of cytochrome $c$ from the mitochondria were all detected, showing that the human Bcl-2 functioned normally in the Bcl-2(P2) cells. Although the apoptotic events tested were identical in the parental cells and transformants, Bcl-2 expression completely failed to inhibit Bph-induced apoptosis in the Bcl-2(P2) cells.

Key words NG108-15 cell; buprenorphine hydrochloride; apoptosis; Bcl-2; mitochondria; mitochondrial apoptotic pathway

The apoptotic "death-signal transduction" pathways are currently classified into three major routes. They are the Fas/FasL pathway, ${ }^{1-4)}$ the mitochondrial pathway, ${ }^{5-8)}$ and the ER (endoplasmic reticulum)-stress activated pathway. ${ }^{9-11)}$ Of these, the mitochondrial route is recognized as fundamental and important, because several apoptosis-inducing stimuli (e.g., intracellular $\mathrm{pH}$ change, certain fatty acids, and anticancer drugs) all share the mitochondrial death-signal cascade. $^{5-8)}$ For example, even in the Fas/FasL apoptosis, a death signal initiated by formation of the DISC (death inducing signaling complex) branches toward the mitochondria to activate caspase-3.,4) Although cytochrome $c$, caspase-9, Apaf-1, ${ }^{12)}$ the Bcl-2 family proteins, ${ }^{13)}$ and Smac/DI$\mathrm{ALBO}^{14,15)}$ are key components in the mitochondrial apoptotic pathway, cytochrome $c$ is thought to be the pivotal molecule in this apoptosis. This is because cytochrome $c$ released from the mitochondria initiates the death-signal cascade of the mitochondrial route; its release is followed by the recruitment of caspase-9 and Apaf-1. ${ }^{12,16,17)}$

Bcl-2-family proteins inhibit apoptosis by blocking the death-signal pathway that starts at the mitochondria. ${ }^{18)}$ Historically, Bcl-2 was found as a protooncogene ${ }^{19)}$; more recently, its over-expression has been shown to protect cells from apoptosis in the mitochondrial pathway. ${ }^{20)}$ Some biochemical studies suggest that Bcl-2 can function both as an ion channel and an adapter or docking protein in the mitochondria $^{21)}$ or endoplasmic reticulum. ${ }^{22)}$ Although the detailed function of Bcl-2 in the mitochondria is still obscure, it may inhibit a voltage-dependent anion channel (VDAC). ${ }^{23)}$ The VDAC may work as a proton pump in response to BH-3only proteins (e.g., Bax, Bim) to release cytochrome $c$ from the mitochondria. ${ }^{24,25)}$ Recently, however, several studies have shown that the over-expression of $\mathrm{Bcl}-2$ does not rescue cells from death in some apoptotic pathways. ${ }^{26-28)}$ These suggest the existence of complicated and unknown apoptotic pathways.

We previously reported that a morphine-derived analgesic, buprenorphine hydrochloride $(\mathrm{Bph})$, induced apoptosis in NG108-15 cells. $^{29,30)}$ NG108-15 is a rodent neuroblastoma $\times$ glioma hybrid cell line. It was generated by Nelson et l. $^{31)}$ in 1976 and has been widely used as an experimental material in pharmacology. In our previous paper, ${ }^{30)}$ we reported that caspase- 3 is activated in this particular apoptosis; however, the detailed apoptotic pathway was still unclear.

In this report, we focused on revealing the details of the Bph-induced apoptotic pathway in NG108-15 cells and show that the mitochondrion is the key organelle in the promotion of this rapid apoptosis. However, the prominent finding was that this particular apoptosis was not suppressed in an NG108-15 transformant that overexpressed Bcl-2.

\section{MATERIALS AND METHODS}

Cells and Culture The NG108-15 cell line used in this study is a rodent-derived neuroblastoma $\times$ glioma hybrid line established by Nelson et al. ${ }^{31)}$ The Bcl-2(P2) cell line is a stable NG108-15 cell transfectant bearing the human Bcl-2 expression vector (pCAGGS bcl-2; a gift from professor Yoshihide Tsujimoto, OSAKA University, Japan). Both lines were routinely cultured at $37^{\circ} \mathrm{C}$ in Dulbecco's Modified Eagle's Medium (Nissui, Japan) supplemented with $10 \%$ fetal bovine serum (FBS), $1 \times 10^{-4} \mathrm{M}$ hypoxanthine, $1 \times 10^{-6} \mathrm{M}$ aminopterin, and $1 \times 10^{-5} \mathrm{M}$ thymidine (normal medium); $100 \mu \mathrm{g} / \mathrm{ml}$ streptomycin was added. Cells were maintained under $100 \%$ humidity with a $5 \% \mathrm{CO}_{2}$ atmosphere. In the serum-starvation condition, NG108-15 and Bcl-2(P2) cells were cultured in a $0.1 \%$ low serum (LS) medium; all of the components of the LS medium were the same as in normal medium except the FBS concentration, which was $0.1 \%$.

Induction of Apoptosis in NG108-15 Cells and Bcl- 
2(P2) Cells To induce apoptosis in the NG108-15 and Bcl2(P2) cells, either Bph was administered or the cells were subjected to serum starvation in $0.1 \%$ LS medium. For Bphinduced apoptosis, a final concentration of $100 \mu \mathrm{M}$ Bph (dissolved in a $40 \%$ methanol solution) was added to the culture medium; 40\% methanol was used as vehicle. For serum starvation, normal medium was replaced with the " $0.1 \%$ LS" medium when the cells were growing logarithmically. Incubation was continued for another $4 \mathrm{~d}$ to induce apoptosis.

Cell Viability, Morphological Observation of Apoptosis, and DNA Fragmentation Assay Cell viability was assayed by the trypan blue dye exclusion method. ${ }^{30)}$ An Olympus model IX-70 inverted phase-contrast microscope equipped with an IX-FLA fluorescence observation device was used to observe the morphological changes in NG108-15 and Bcl2(P2) cells. Typical nuclear condensation was used as an apoptotic marker and observed using Hoechst 33258 dye (Molecular Probes, U.S.A.). An assay of DNA ladder formation over time was conducted as described previously. ${ }^{29)}$

Flow Cytometric Analysis for the Progression of the Loss of Mitochondrial Transmembrane Potential To detect changes in the mitochondrial membrane potential, we used the ApoAlert ${ }^{\circledR}$ Mitochondrial Membrane Sensor Kit (BD Biosciences Clontech, U.S.A.) following the manufacturer's instructions. In brief, intact or apoptotic NG108-15 and Bcl-2(P2) cells were harvested, washed with ice-cold PBS (-), and prepared at a final density of $10^{9}$ cells per $\mathrm{ml}$ in a flow cytometry tube. The cells were spun and resuspended in $1 \mathrm{ml}$ of diluted Mitosensor ${ }^{\circledR}$ reagent, which came with the kit. After a $15-\mathrm{min}$ incubation in a $\mathrm{CO}_{2}$ incubator, $1 \mathrm{ml}$ of Incubation buffer (from the kit) was added, and the cell suspension was prepared for flow cytometry (Coulter, EPICS ${ }^{\circledR}$ XL, U.S.A.). Aggregated Mitosensor ${ }^{\circledR}$ dye was detected in the PI channel and monomeric Mitosensor ${ }^{\circledR}$ was detected in the fluorescein isothiocyanate (FITC) channel.

Western Blotting To detect cytochrome $c$ and caspase-9, intact or apoptotic NG108-15 or Bcl-2(P2) cells were harvested by trypsinization at the sampling times indicated in each figure; the cells were then washed with ice-cold PBS (-). The cell fractionation to obtain the mitochondrial and cytosolic fractions was conducted using the ApoAlert ${ }^{\circledR}$ Cell Fractionation Kit (BD Biosciences Clontech, U.S.A.) following the manufacturer's instructions. Briefly, the cell pellet was suspended in the Cell Fractionation Buffer with protease inhibitors (all reagents were from the kit) and spun first at $700 \times \boldsymbol{g}$ for $5 \mathrm{~min}$ at $4{ }^{\circ} \mathrm{C}$, then at $10000 \times \boldsymbol{g}$ for $25 \mathrm{~min}$ at $4{ }^{\circ} \mathrm{C}$. The pellet obtained after the second centrifugation step was the mitochondrial fraction, and it was resuspended in the Cell Fractionation Buffer. The supernatant from the second centrifugation step was used as the cytosolic fraction.

For the western blotting of cytochrome $c, 10 \mu \mathrm{g}$ each of mitochondrial and cytosolic protein was subjected to $15 \%$ SDS-polyacrylamide gel electrophoresis (SDS-PAGE). A 10$\mu \mathrm{g}$ aliquot of protein from the cytosolic fraction was used for the detection of capsase- 9 and separated by $12.5 \%$ SDSPAGE. Proteins were then electro-transferred to a PROTRAN $^{\circledR}$ nitrocellulose transfer membrane (Schleicher \& Schull, U.S.A.). The membrane was blocked in $10 \%$ non-fat milk in TBST buffer ( $20 \mathrm{~mm}$ Tris- $\mathrm{HCl}, 0.5 \mathrm{~m} \mathrm{NaCl}, 0.05 \%$ Tween 20, pH 7.5) at room temperature for $1 \mathrm{~h}$. The primary antibodies were as follows: rabbit anti mouse-cytochrome $c$ antibody (diluted 1:100; came with the ApoAlert ${ }^{\circledR}$ Cell Fractionation Kit, BD Biosciences Clontech), mouse anti mouse-caspase-9 antibody (diluted $1: 100$; MBL, Japan).

A $10-\mu \mathrm{g}$ aliquot of mitochondrial protein was also subjected to $12.5 \%$ SDS-PAGE to detect the human Bcl-2 protein expressed in the mitochondria. For this purpose, we used intact NG108-15 cells, mock-vector (pCAGGS)-transfected NG108-15 cells, and Bcl-2(P2) cells as follows. The individual NG108-15 transfectants were assayed by western blotting to determine the amount of Bcl-2 expressed. The primary antibodies were a mouse anti-human Bcl-2 monoclonal antibody (diluted 1:200; NEO MARKERS, U.S.A.) or mouse anti-mouse Bcl-2 monoclonal antibody (diluted 1 : 500; NEO MARKERS, U.S.A.), the latter of which was used as a control to detect the intrinsic mouse Bcl-2 protein. Beta-actin antibody (diluted 1:5000; CHEMICON, U.S.A.) and cytochrome $c$ oxidase subunit IV (COX4) antibody (diluted 1 : 100; BD Biosciences Clontech, U.S.A.) were used respectively to detect $\beta$-actin and COX4 as loading controls for the western blots. All the western blot procedures to detect $\beta$ actin and COX4 were as described above.

Immunofluorescence Microscopy $\mathrm{Bcl}-2(\mathrm{P} 2)$ cells were harvested and fixed in a 3.7\% formaldehyde-PBS $(+)$ solution. The collected cells were incubated in $0.1 \%$ Triton X100-PBS $(+)$. The primary antibodies (human or mouse anti-Bcl-2 monoclonal antibodies, NEO MARKERS, U.S.A.; mouse COX4 antibody, BD Biosciences Clontech, U.S.A.) were diluted $1: 100$ in $2 \%$ BSA-PBS $(+)$ and incubated with the cells at $4{ }^{\circ} \mathrm{C}$ for $16 \mathrm{~h}$. The FITC/rhodamine-conjugated secondary antibodies (Santa Cruz Biotechnology, U.S.A.) were used at a $1: 100$ dilution in $2 \%$ BSA-PBS $(+)$ solution, and the incubation was for $1 \mathrm{~h}$ at room temperature. The cells were mounted using the ProLong ${ }^{\circledR}$ Antifade Kit (Molecular Probe, U.S.A.), and an Olympus FV-500 laser confocal microscope was used to observe the cellular localization of cytochrome $c$ and $\mathrm{Bcl}-2$.

Colorimetric Assay for Caspase-3 Activation Caspase3 activation following treatment with $100 \mu \mathrm{M}$ Bph in NG10815 and Bcl-2(P2) cells was assayed using the ApoAlert ${ }^{\circledR}$ Caspase Colorimetric Assay Kit (BD Biosciences Clontech, U.S.A.), following the manufacturer's instructions. The increase in the caspase- 3 activity was determined by comparing the results with uninduced control samples; that is, we subtracted the background reading from cell lysates and buffers from the reading of the induced and uninduced cells, before calculating the fold increase in the caspase activities per $\mu \mathrm{g}$ protein per $\mathrm{ml}$ cell lysate.

\section{RESULTS}

The Death Signal of Bph-Induced Apoptosis is Routed through the Mitochondria One of the major death-signal transduction pathways is known to cascade from the mitochondria to caspase-3, the effector molecule. To determine the detailed signal-transduction pathway of the Bph-induced apoptosis in NG108-15 cells, we studied the contribution of several representative molecules to the mitochondrial pathway: cytochrome $c$, caspases-9, and -3 (Fig. 1).

As the first step, we measured the loss of mitochondrial membrane potential, because this loss is known usually to occur in advance of the release of cytochrome $c$ from the mi- 
A

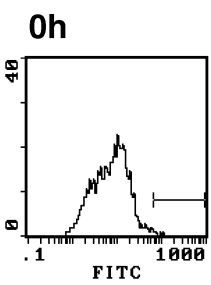

$2 \mathrm{~h}$

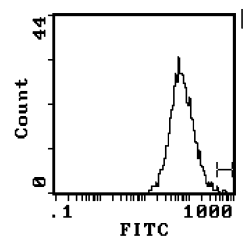

C

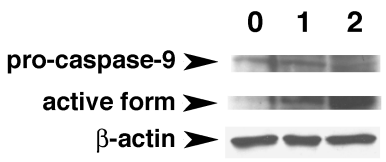

D

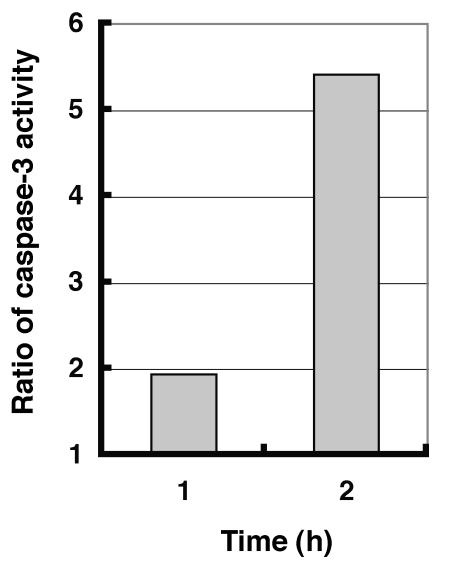

Fig. 1. Time-Dependent Loss of Mitochondrial Membrane Potential, Cytochrome $c$ Release, and Caspase-9 and -3 Activation in NG108-15 Cells Undergoing Bph-Induced Apoptosis

(A) Flow cytometric analysis of the time course of the loss of mitochondrial membrane potential after Bph administration in NG108-15 cells. The cells were stained with a cationic dye called MitoSensor $^{\mathrm{TM}}$ (BD Biosciences Clontech, U.S.A.), which fluoresces differently in apoptotic and nonapoptotic cells. Histograms of apoptotic NG108-15 cells are shown. (B) Western analysis of cytochrome $c$ released from mitochondria after Bph treatment. Bph $(100 \mu \mathrm{M})$ was added to the NG108-15 cell medium at time 0 h. Cells were harvested at the times (hours) listed above each lane. COX4 and $\beta$-actin were used as loading controls for the western blot analysis. (C) Pro-caspase-9 was activated in NG108-15 cells after Bph was administered. Sampling times are listed above each lane. The active form of caspase-9 is a truncated 37-kD peptide. Beta-actin was used as a loading control for the western blot analysis. (D) Results from a colorimetric assay for caspase-3 activity. The X-axis gives hours after Bph administration. The Y-axis gives the fold change in caspase-3 activity. At $0 \mathrm{~h}$ (right after Bph administration) the activity of caspase- 3 was defined as " 1. "

tochondria. ${ }^{32,33)}$ Using flow cytometric analysis (FACS), we observed that the loss of mitochondrial membrane potential was already induced by $1 \mathrm{~h}$ after the administration of Bph to NG108-15 cells (Fig. 1A). An increase in the number of FITC $(+)$ cells, which indicated the disruption of the mitochondrial membrane structure, was also seen as early as $1 \mathrm{~h}$ after Bph administration in NG108-15 cells. These data strongly suggested that rapid disruption of the mitochondria was initiated by the administration of Bph to NG108-15 cells.

We next investigated the release of cytochrome $c$ from mitochondria using western blotting and found that it also started by $1 \mathrm{~h}$ after Bph administration to the NG108-15 cells (Fig. 1B). In agreement with this finding, the cytochrome $c$ concentration increased with time in the cytosolic fraction (Fig. 1B)

Once cytochrome $c$ is released from the mitochondria, it triggers the activation of pro-caspase- 9 in cooperation with Apaf-1. ${ }^{12)}$ The activated death signal then cascades downstream to pro-caspase-3. Activated caspase- 3 finally plays the important role of transmitting the death signal to a final death executor, one or more DNases such as CAD and ICAD. ${ }^{34,35)}$ We assessed the activation of caspase- 9 and -3 by western blotting and a colorimetric method, and found that the activation of these caspases started as early as $1 \mathrm{~h}$ after Bph-administration, and it proceeded in a time-dependent manner (Figs. $1 \mathrm{C}, \mathrm{D})$. These results strongly suggested that the Bph-in- duced death signal is routed through the mitochondrial apoptotic pathway in NG108-15 cells.

Construction of Bcl-2 Stable Transfectants and the Cellular Localization of Bcl-2 The mitochondrial apoptotic pathway is usually inhibited by the over-expression of Bcl$2 .^{13,18)}$ To confirm the results obtained in Fig. 1, we constructed stable Bcl-2-expressing NG 108-15 transfectants with a Bcl-2 expression vector, pCAGGS bcl-2, which contains the human Bcl-2 open reading frame. Seven transfectants were individually isolated, and their level of Bcl-2 expression was assessed by western blotting (Fig. 2A). Of these seven clones, the "P2" clone expressed the most Bcl-2, and therefore we chose it for use in further experiments and renamed it Bcl-2(P2).

We next explored the cellular localization of the Bcl-2 protein expressed in Bcl-2(P2) cells using in vitro cell fractionation (Fig. 2B) and immunofluorescence microscopy (Fig. 2C).

Intact NG108-15 cells, Bcl-2(P2) cells, and vector (pCAGGS)-transfected NG108-15 cells were harvested, and the mitochondrial and cytosolic fractions were separated. Western blots showed that the human Bcl-2 protein was expressed and localized to the mitochondrial fraction of only the Bcl-2(P2) cells (Fig. 2B, lane 2). We also detected a smaller amount of the Bcl-2 protein in the cytosolic fraction of the Bcl-2(P2) cells (Fig. 2B, lane 5), which may represent over-expressed human Bcl-2 in the endoplasmic reticulum, 
A

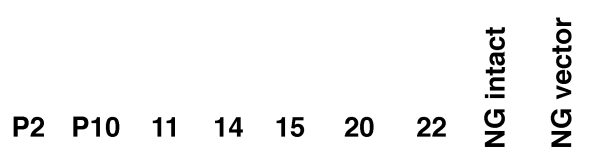

hBcl-2
B

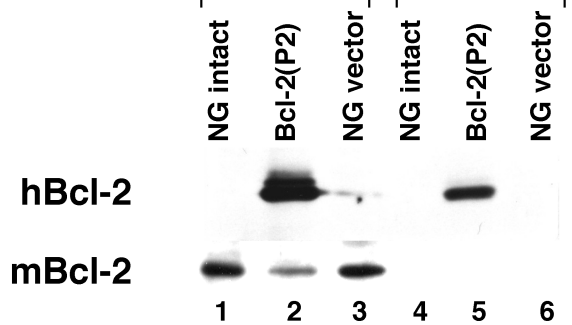

a

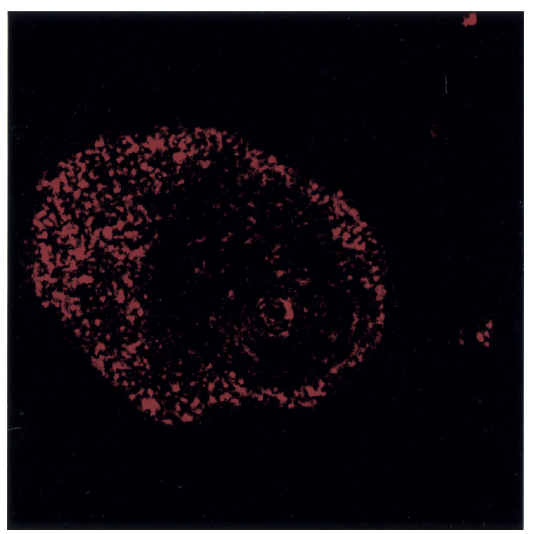

b

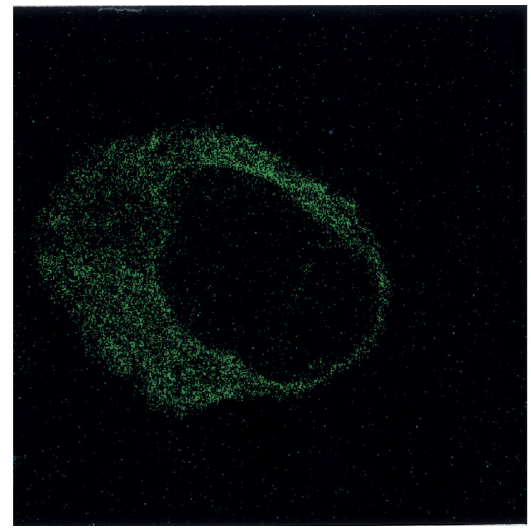

C

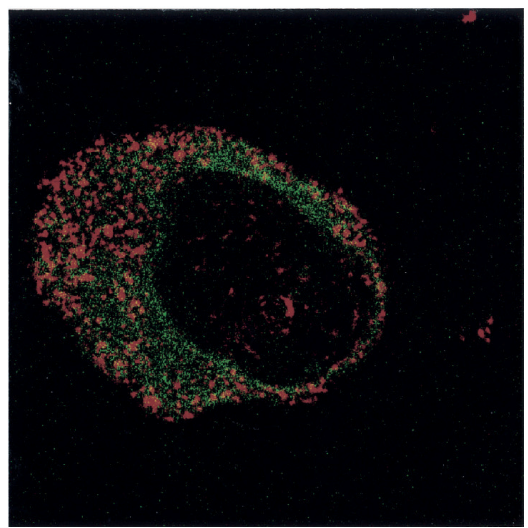

Fig. 2. Establishment of Bcl-2-Over-Expressing NG108-15 Transfectants and Their Characterization

(A) The expression of human Bcl-2 (hBcl-2) in various transfectants was evaluated by western blotting. The numbers above the western signals are the clone names. Clone $\mathrm{P} 2$ had the highest Bcl-2 expression, so it was named Bcl-2(P2) and used for further experiments. Clones 15 and 11 were also used as middle and low-level Bcl-2 expressors in the experiments shown in Fig. 4. "NG intact" and "NG vector" refer to parental NG108-15 cells and empty vector (pCAGGS)-transfected NG108-15 cells, respectively. (B) Subcellular localization of the Bcl-2 protein. Mitochondrial and cytosolic fractions were prepared from NG intact, NG vector, and Bcl-2(P2) cells, respectively. The cells were grown and the subcellular localization of the Bcl-2 protein was detected on western blots. Lanes 1 through 3 show mitochondrial fractions and 4 through 6 show cytosolic fractions. Samples in lanes 1 and 4 were prepared from intact NG intact cells, lanes 2 and 5 from Bcl-2(P2) cells, and lanes 3 and 6 from NG vector cells, respectively. The upper panel shows the western data using the hBcl-2 first antibody. The lower panel shows data obtained using the mouse Bcl-2 (mBcl-2) first antibody. (C) Localization of the Bcl-2 protein using laser confocal immunofluorescence microscopy. The COX4 protein (which localizes to mitochondria) is visualized as red color in the left panel (a). The center panel (b) shows the distribution of the Bcl-2 protein in green. The right panel (c) shows the left and the center images merged. Magnification $=$ original $\times 1000$.

as was previously reported by Krajewski et l. $^{36)}$ In the same experiment, the intrinsic mouse Bcl-2 expression was also examined using an anti-mouse Bcl-2 antibody, and we confirmed that the intrinsic mouse Bcl-2 protein was mostly localized to the mitochondrial fraction.

To obtain further evidence that Bcl-2 expression was predominantly in the mitochondria in the Bcl-2(P2) cells, we performed immunofluorescence microscopy using the mouse COX4 antibody to label the mitochondria and human antiBcl-2 antibodies (Fig. 2C). The COX4 labeling appeared as red fluorescent spots scattered throughout the cytoplasm (Fig. 2C-a); Bcl-2 labeling appeared as green fluorescent spots distributed ubiquitously in the cytosol, although some more-intense staining was observed in the nuclear membrane and the exo-cytosolic space (Fig. 2C-b). The pictures of the COX4 and Bcl-2 labeling were merged, and the merged image showed that the Bcl-2 protein was predominantly expressed in the mitochondria of Bcl-2(P2) cells (yellow spots); however, considerable Bcl-2 protein was clearly observed in places other than in the mitochondria (Fig. 2C-c). Krajewski et al., Hacki et al., and Ako et al. ${ }^{36-38)}$ reported that the Bcl2 protein is located outside the mitochondrion, and the green spots in the merged picture probably represent the expression of the Bcl-2 protein in the endoplasmic reticulum and nuclear envelope.
The Over-Expressed Bcl-2 Protein Functions Normally as an Anti-apoptotic Protein in Bcl-2(P2) Cells We previously showed that a different kind of apoptosis was induced by serum-starvation in NG108-15 cells, ${ }^{29)}$ and here we used this apoptotic system to evaluate the function of the overexpressed $\mathrm{Bcl}-2$ protein as an apoptosis inhibitor in $\mathrm{Bcl}-2(\mathrm{P} 2)$ cells. First, we grew NG108-15 and Bcl-2(P2) cells under serum-starved conditions, and followed their viability for the next five days (Fig. 3A). The viability of the serum-starved NG108-15 cells diminished slowly, and no viable cells were observed on day 4 of the experiment. In contrast, most of the Bcl-2(P2) cells were viable up to day 3 and 50\% were still living on day 4 (Fig. 3A). Unlike this serum-starvation-induced cell death, we had previously found that Bph-induced cell death progressed rapidly in NG108-15 cells, with the viability reaching zero about 6 to $8 \mathrm{~h}$ after the Bph administration. $^{29)}$

We next looked for DNA ladder formation, which is a characteristic feature of most kinds of apoptosis. A DNA ladder was observed as early as two days after the initiation of serum-starvation in the parental NG108-15 cells. In contrast, the Bcl-2(P2) cells showed almost no DNA-ladder formation during the five days of the experiment (Fig. 3B).

We further assessed cytochrome $c$ release from the mitochondrial fraction into the cytosol in serum-starved NG108- 
A

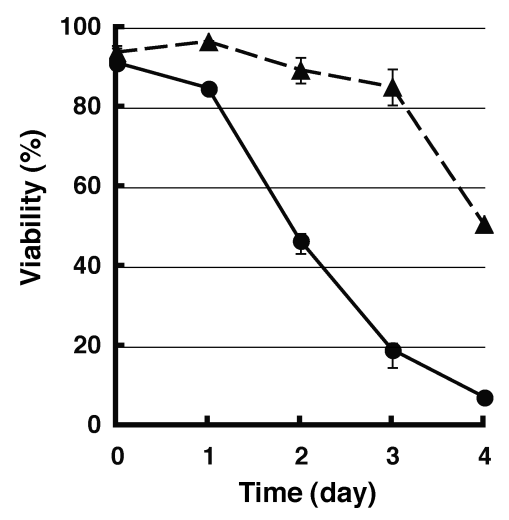

C

NG108-15

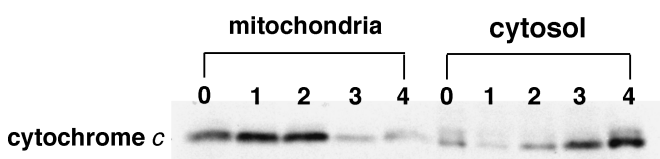

B

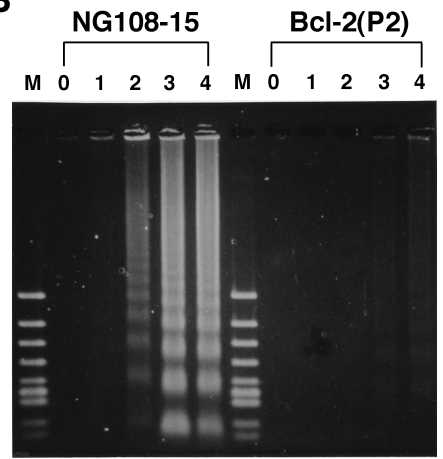

Fig. 3. The Over-Expression of Bcl-2 Suppressed the Serum Starvation-Induced Apoptosis of NG108-15 Cells

(A) Viability of NG108-15 and Bcl-2(P2) cells. The closed circle with solid lines and the closed triangle with broken lines indicate the viability of the NG108-15 cells and Bcl2(P2) cells, respectively. On day 0 , the cells were placed under serum-starvation conditions, and they were harvested every $24 \mathrm{~h}$ thereafter (day 1 through day 4 ). Their viability was assessed using the trypan blue dye exclusion method. (B) Results of the DNA fragmentation assay during serum starvation. DNA fragmentation was assessed on days 0 - 4 . The cell lines and sampling times are shown above the lanes. (C) The release of cytochrome $c$ is inhibited in serum-starved Bcl-2(P2) cells. Shown are western blots of NG108-15 cells probed with an anti-cytochrome $c$ antibody and of Bcl-2(P2) cells that were harvested every $24 \mathrm{~h}$ from day 0 (the day serum-starvation was begun) through day 4 and separated into mitochondrial and cytosolic fractions. These are representative data from three independent experiments.

15 and Bcl-2(P2) cells (Fig. 3C). As we expected, time-dependent cytochrome $c$ release was observed in the NG108-15 cells (Fig. 3C). In contrast, the western blotting data clearly showed that the efflux of cytochrome $c$ was completely inhibited in the Bcl-2(P2) cells.

These results demonstrated that the serum-starvation-induced apoptosis in NG108-15 cells was an effective positive control, showing that the over-expressed Bcl-2 protein functioned normally in the $\mathrm{Bcl}-2(\mathrm{P} 2)$ cells as an apoptosis inhibitor.

Over-Expression of Bcl-2 in NG108-15 Cells Never Inhibits Bph-Induced Apoptosis Using Bcl-2(P2) cells, we conducted a variety of experiments to test whether the overexpressed Bcl-2 also inhibited the apoptotic events triggered by Bph in the parental cells. The experiments were: a cell viability test, a DNA fragmentation assay, morphological observation of nuclear condensation, FACS analysis for the loss of mitochondrial membrane potential, cytochrome $c$ release from mitochondria, and caspase- 9 and -3 activation (Figs. $4 \mathrm{~A}-\mathrm{G})$.

We found that the Bcl-2(P2) cells never survived long after the administration of $\mathrm{Bph}$, and that, in fact, the viability curves for the NG108-15 and Bcl-2(P2) cells almost coincided (Fig. 4A). The DNA fragmentation assay and the morphological observation of nuclear condensation also clearly indicated that the Bcl-2(P2) cells died of apoptosis, as did the parental strain, NG108-15. As early as $2 \mathrm{~h}$ after Bph administration, a clear and distinct DNA ladder appeared (Fig. 4B). In addition, Hoechst 33258 staining indicated obvious and typical nuclear condensation in the Bcl-2(P2) cells at $2 \mathrm{~h}$ (Fig. 4C). These results show that over-expression of Bcl-2 failed to suppress the Bph-induced apoptosis in Bcl-2(P2) cells.

The results shown in Figs. 4D-G elaborated on the finding that the over-expression of Bcl-2 had no effect on the mitochondrial death-signal transduction pathway in this system. The loss of the mitochondrial membrane potential started by $1 \mathrm{~h}$ after Bph administration (Fig. 4D), as it did in the parental NG108-15 cells shown in Fig. 1A. The time-dependent release of cytochrome $c$ from mitochondria (Fig. 4E) followed by the activation of caspases-9, and -3 (Figs. 4F, G) was observed in the Bcl-2(P2) cells, and the results were just as in NG108-15 cells (Fig. 1). We also repeated the experiments shown in Fig. 4A-G with two other Bcl-2-expressing NG108-15 transfectants, clones "15" and "11" (see Fig. 2A). The 15 and 11 cells were middle- and low-level Bcl-2 expressors, and their responses to Bph treatment (data not shown) were almost the same as those of the Bcl-2(P2) cells, shown in Figs. $4 \mathrm{~A}-\mathrm{G}$.

\section{DISCUSSION}

Here we studied the apoptotic pathway induced by Bph in the NG108-15 cell line. One characteristic feature of this pathway is its rapidity. Less than $2 \mathrm{~h}$ after the administration of Bph to NG108-15 cells, the shape of the cells drastically changes from that of typical neural cells to simple round shapes, and the cells start to float in the medium. Loss of viability occurs almost simultaneously with the drastic morphological changes, as do two other characteristic features, nuclear condensation and DNA-ladder formation. ${ }^{29,30)} \mathrm{We}$ found a paper reporting ultra-rapid apoptosis, ${ }^{39)}$ in which cells start dying within 2 min of drug administration. In most cases, however, studies report that drug-induced cell-death 
A

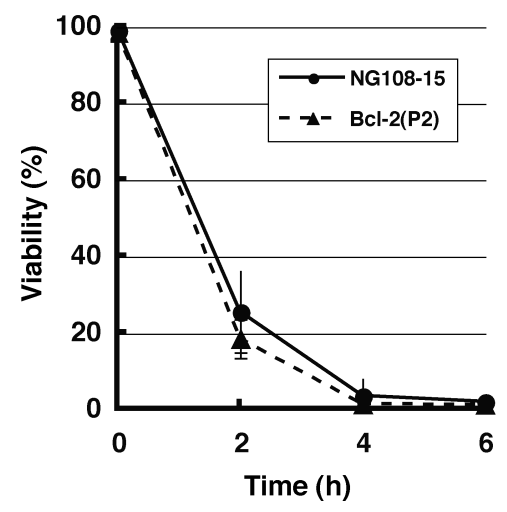

D
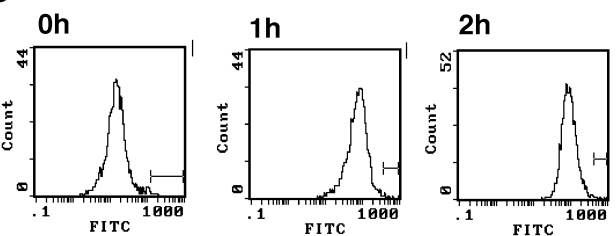

E

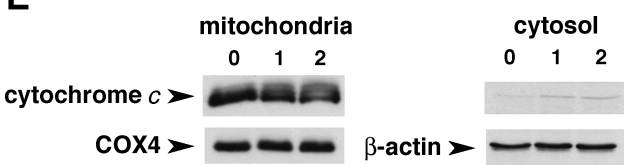

C

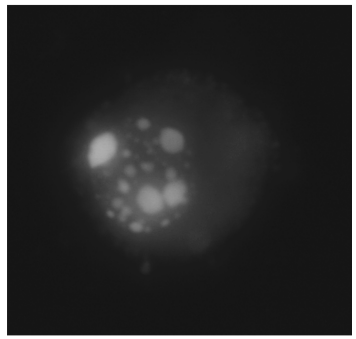

F

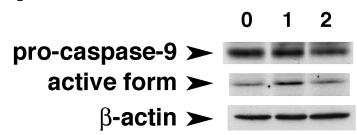

$\beta$-actin

G

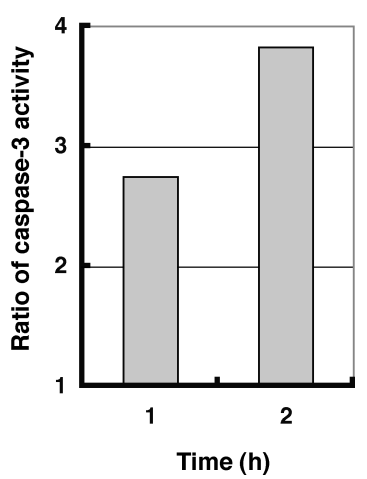

Fig. 4. The Over-Expression of Bcl-2 in NG108-15 Cells Failed to Inhibit the Apoptosis Induced by Bph

(A) The viability of NG108-15 and Bcl-2(P2) cells was compared using the trypan blue dye exclusion method. Closed circles with a solid line and closed triangles with a broken line indicate the viability of the NG108-15 and Bcl-2(P2) cells, respectively. (B) DNA fragmentation assay for parental NG 108-15 cells and Bcl-2(P2) cells after Bph administration. At the 0-, 2-, and 4-h sampling times, both NG108-15 and Bcl-2(P2) cells were harvested, and the fragmented DNA was separated by electrophoresis. DNA size markers from a $\phi X-H i n c$ II digest are shown in the left lane (M). (C) Fluorescence micrograph of Hoechst 33258-stained Bcl-2(P2) cells two hours after Bph administration. Magnification $=$ original $\times 400$. (D) Flow cytometric analysis of the time course of the loss of mitochondrial membrane potential after Bph-administration in Bcl-2(P2) cells. (E) Release of cytochrome $c$ from the mitochondria into the cytosolic fraction after Bph administration. COX 4 and $\beta$-actin were used as a loading control for the western blot analysis. (F) Activation of pro-caspase-9 in Bcl-2(P2) cells after Bph administration. (G) Activation of caspase-3 in Bcl-2(P2) cells after Bph administration. The procedures for the experiments shown in panels (D, E, F, and G) are the same as for those shown in Fig. 1.

occurs over several days following drug administration. ${ }^{40,41)}$ In this context, this Bph-induced apoptosis in NG108-15 cells belongs in the category of "rapid apoptosis," and the mechanism of this particular apoptotic pathway drew our scientific interest.

In our previous study, ${ }^{30)}$ we reported that caspase- 3 activation occurs as a consequence of this particular apoptosis. However, caspase-3 plays a pivotal role in many apoptotic pathways, including the Fas/FasL pathway, the mitochondrial pathway, and the endoplasmic reticulum stress pathway. Therefore, we initiated the present study to identify the signal-transduction pathway of this particular apoptosis.

We focused on the role of three molecules that are important in the major mitochondrial apoptotic pathway: cytochrome $c$ and capsase- 9 and -3 . We also observed the loss of the mitochondrial membrane potential. Within in $1 \mathrm{~h}$ after the administration of Bph, the biochemical events representing the mitochondrial apoptotic route, the loss of the mitochondrial membrane potential, the efflux of cytochrome $c$ from the mitochondria, and the activation of caspase- 9 and -3 were all observed in our apoptosis system. The cytochrome $c$ efflux and capase activation that occurred within $1 \mathrm{~h}$ after Bph administration were not clearly observed, probably because the amount of the cytochrome $c$ released and caspase- 9 activated were too small to be detected by western blot. However, the loss of mitochondrial membrane potential was clearly observed by $1 \mathrm{~h}$ after Bph administration and was prolonged until $2 \mathrm{~h}$. These results strongly suggested that the very first event of this apoptotic pathway was the disruption of the mitochondrial membrane function by Bph, and the fol- 
lowing apoptotic events on the mitochondrial route occurred almost simultaneously. Therefore, it seemed likely that this Bph-induced apoptosis involved the mitochondrial death-signal transduction pathway.

The importance of the mitochondria as a death-signal generator or mediator has been widely accepted. Bcl-2 family proteins, whether pro- or anti-apoptotic, make contact with the mitochondria during apoptotic events. ${ }^{42-44)}$ Also, the mitochondrial protein Smac/DIALBO is released from the mitochondria by some apoptotic stimuli, including UV-radiation, but the subsequent caspase activation is blocked by Bcl2 over-expression. ${ }^{45}$ Because we obtained evidence that our Bph-induced apoptosis cascaded from the mitochondria, as shown in Fig. 1, we examined the contribution of the Bcl-2 protein as a representative anti-apoptotic protein of this apoptotic pathway and established NG108-15 transformant lines that expressed the human $\mathrm{Bcl}-2$ protein (Fig. 2).

Using one of these lines, Bcl-2(P2), we observed that the human $\mathrm{Bcl}-2$ protein was predominantly in the mitochondria, although there was some expression in the cytosol (probably in the endoplasmic reticulum) and the nuclear membrane (Figs. 2B, 2C-b). The endogenous mouse $\mathrm{Bcl}-2$ protein was expressed predominantly in the mitochondrial fraction of intact NG108-15 cells, empty vector-transfected NG108-15 cells, and Bcl-2(P2) cells. However, the intrinsic expression of mouse Bcl-2 in the cytosol fraction was very faint and beyond the sensitivity of our western blots. These data indicated that the exogenous human Bcl-2 protein was expressed normally in the NG108-15 cells. However, we needed further confirmation that the overexpressed $\mathrm{Bcl}-2$ protein functioned as an apoptosis inhibitor in the Bcl-2(P2) cells. For this purpose, we exploited another apoptotic pathway in NG108-15 cells that is induced by serum-starvation. The viability curve of $\mathrm{Bcl}-2(\mathrm{P} 2)$ cells, the result of the DNA-ladder formation assay in Figs. 3A, 3B, and the cytochrome $c$ efflux data (Fig. $3 \mathrm{C})$ clearly indicated that the over-expressed $\mathrm{Bcl}-2$ protein functioned normally in Bcl-2(P2).

Apoptotic pathways are diverse, and include rare forms such as apoptosis without DNA-ladder formation, ${ }^{46)}$ caspaseindependent apoptosis, ${ }^{47)}$ and $\mathrm{Bcl}-2$ independent apoptosis. ${ }^{48)}$ Contrary to our expectations, we observed that the over-expressed human Bcl-2 did not rescue the Bph-induced apoptosis in Bcl-2(P2) cells (Fig. 4). Rather, all the apoptotic events tested were identical in the Bcl-2(P2) cells and the parental NG108-15 cells. Taken together, these results lead us to conclude that the Bph-induced apoptotic pathway is routed through the mitochondria, but does not respond to $\mathrm{Bcl}-2$ over-expression.

In this report, we have presented an unusual mitochondrial apoptotic pathway. The most prominent feature of this particular apoptosis is that some unknown function of Bph cancelled the anti-apoptotic activity of Bcl-2. This may be owing to some physico-chemical characteristic of Bph, because Bph is a hydrophobic compound and easily passes through the double lipid layers of biomembranes. Even if this is true, there is another question that remains: How does Bph disrupt the function of $\mathrm{Bcl}-2$ as an anti-apoptotic protein in the mitochondria? Thus, we need to investigate the possibility that proteins on the mitochondrial outer membrane interact with $\mathrm{Bph}$ and transduce its apoptotic signal. Otherwise, it is difficult to explain how Bph could pass through the cell mem- branes without causing problems, and damage only the mitochondrial membrane. Further study based on a proteome profiling system might help to identify the other players in this new apoptotic pathway.

Acknowledgements This work was supported in part by a Nihon University General Individual Research Grant for 2001, and 2003 (to F. K.) and A Grant from the Ministry of Education, Culture, Sport, Science, and Technology of Japan to promote multi-disciplinary research projects. (to F. K., A. U., and M. A.)

\section{REFERENCES}

1) Kavurma M. M., Khachigian L. M., Cell Death Differ., 10, 36-44 (2003).

2) Peter M. E., Krammer P. H., Cell Death Differ., 10, 26-35 (2003).

3) Wajant H., Nature (London), 296, 1635-1636 (2002).

4) Scaffidi C., Fulda S., Srinivasan A., Friesen C., Li F., Tomaselli K. J., Debatin K.-M., Krammer P. H., Peter M. E., EMBO J., 17, 16751687 (1998).

5) Hirpara J. L., Clément M.-V., Pervaiz S., J. Biol. Chem., 276, 514 $521(2001)$

6) Rebbaa A., Chou P. M., Emran M., Mirkin B. L., Cancer Chemother Pharmacol., 48, 423-428 (2001).

7) Tournier C., Hess P., Yang D. D., Xu J., Turner T. K., Nimnual A., BarSagi D., Jones S. N., Flavell R. A., Davis R. J., Science, 288, 870874 (2000).

8) Wieckowski M. R., Brdiczka D., Wojtczak L., FEBS Lett., 484, 6164 (2000)

9) Breckenridge D. G., Germain M., Mathai J. P., Nguyen M., Shore G. C., Oncogene, 22, 8608-8618 (2003).

10) Adams J. M., Genes Dev., 17, 2481-2495 (2003).

11) Matsuzawa A., Nishitoh H., Tobiume K., Takeda K., Ichijo H., Antioxid. Redox Signal., 4, 415-425 (2002).

12) Li P., Nijhawan D., Budihardjo I., Srinivasula S. M., Ahmad M., Alnemri E. S., Wang X., Cell, 91, 479-489 (1997).

13) Reed J. C., Nature (London), 387, 773-776 (1997).

14) Du C., Fang M., Li Y., Li L., Wang X., Cell, 102, $33-42$ (2000).

15) Verhagen A. M., Ekert P. G., Pakusch M., Silke J., Connolly L. M., Reid G. E., Moritz R. L., Simpson R. J., Vaux D. L., Cell, 102, 43-53 (2000).

16) Zou H., Li Y., Liu X., Wang X., J. Biol. Chem., 274, 11549-11556 (1999).

17) Reed J. C., Cell, 91, 559-562 (1997).

18) Tsujimoto Y., Shimizu S., FEBS Lett., 466, 6-10 (2000).

19) Tsujimoto Y., Cossman J., Jaffe E., Croce C. M., Science, 228, 1440 1443 (1985).

20) Kluck R. M., Bossy-Wetzel E., Green D. R., Newmeyer D. D., Science, 275, 1132-1136 (1997).

21) Heiden M. G. V., Chandel N. S., Williamson E. K., Schumacker P. T., Thompson C. B., Cell, 91, 627-637 (1997).

22) Foyouzi-Youssefi R., Arnaudeau S., Borner C., Kelly W. L., Tschopp J., Lew D. P., Demaurex N., Krause K.-H., Proc. Natl. Acad. Sci. U.S.A., 97, 5723-5728 (2000).

23) Shimizu S., Narita M., Tsujimoto Y., Nature (London), 399, 483-487 (1999).

24) Sugiyama T., Shimizu S., Matsuoka Y., Yoneda Y., Tsujimoto Y., Oncogene, 21, $4944-4956$ (2002).

25) Shimizu S., Matsuoka Y., Shinohara Y., Yoneda Y., Tsujimoto Y., J. Cell. Biol., 152, 237-250 (2001).

26) Achenbach T. V., Müller R., Slater E. P., J. Biol. Chem., 275, 32089 32097 (2000)

27) Keogh S. A., Walczak H., Bouchier-Hayes L., Martin S. J., FEBS Lett., 471, 93-98 (2000).

28) Huang D. C. S., Hahne M., Schroeter M., Frei K., Fontana A., Villunger A., Newton K., Tschopp J., Strasser A., Proc. Natl. Acad. Sci. U.S.A., 96, 14871-14876 (1999).

29) Kugawa F., Arae K., Ueno A., Aoki M., Eur. J. Pharmacol., 347, 105-112 (1998)

30) Kugawa F., Ueno A., Aoki M., Biol. Pharm. Bull., 23, 930-935 
(2000)

31) Nelson P., Christian C., Nirenberg M., Proc. Natl. Acad. Sci. U.S.A., 73, 123-127 (1976).

32) Kantrow S. P., Piantadosi C. A., Biochem. Biophys. Res. Commun., 232, 669-671 (1997).

33) Kantrow S. P., Tatro 1. G., Piantadosi C. A., Free Radic. Bio. Med., 28, $251-260(2000)$

34) Enari M., Sakahira H., Yokoyama H., Okawa K., Iwamatsu A., Nagata S., Nature (London), 391, 43-50 (1998).

35) Sakahira H., Enari M., Nagata S., Nature (London), 391, 96-99 (1998).

36) Krajewski S., Tanaka S., Takayama S., Schibler M. J., Fenton W., Reed J. C., Cancer Res., 53, 4701-4714 (1993).

37) Hacki J., Egger L., Monney L., Conus S., Rosse T., Fellay I., Borner C., Oncogene, 19, 2286-2295 (2000).

38) Akao Y., Otsuki Y., Kataoka S., Ito Y., Tsujimoto Y., Cancer Res., 54, 2468-2471 (1994).

39) Fladmark K. E., Brustugun O. T., Hovland R., Bøe R., Gjertsen B. T., Zhivotovsky B., Døskeland S. O., Cell Death Differ., 6, 1099-1108
(1999).

40) Dirsch V. M., Stuppner H., Vollmar A. M., Cancer Res., 61, 58175823 (2001).

41) Wieder T., Essmann F., Prokop A., Schmelz K., Schulze-Osthoff K., Beyaert R., Dörken B., Daniel P. T., Blood, 97, 1378-1387 (2001).

42) Huang D. C. S., Strasser A., Cell, 103, 839-842 (2000).

43) Pawlowski J., Kraft A. S., Proc. Natl. Acad. Sci. U.S.A., 97, 529-531 (2000).

44) Luo X., Budihardjo I., Zou H., Slaughter C., Wang X., Cell, 94, 481490 (1998).

45) Adrain C., Creagh E. M., Martin S. J., EMBO J., 20, 6627-6636 (2001).

46) Zhang J., Wang X., Bove K. E., Xu M., J. Biol. Chem., 274, 3745037454 (1999)

47) Nylandsted J., Rohde M., Brand K., Bastholm L., Elling F., Jäättelä M., Proc. Natl. Acad. Sci. U.S.A., 97, 7871-7876 (2000).

48) Chaloupka R., Petit P. X., Israë1 N., Sureau F., FEBS Lett., 462, 295301 (1999). 